\author{
Anna Maria Brambilla \\ Stefano Aliberti \\ Elena Prina \\ Francesco Nicoli \\ Manuela Del Forno \\ Stefano Nava \\ Giovanni Ferrari \\ Francesco Corradi \\ Paolo Pelosi \\ Angelo Bignamini \\ Paolo Tarsia \\ Roberto Cosentini
}

\section{Erratum to: Helmet CPAP vs. oxygen therapy in severe hypoxemic respiratory failure due to pneumonia}

Published online: 27 May 2014

(C) Springer-Verlag Berlin Heidelberg and ESICM 2014

The online version of the original article can be found under doi: 10.1007/s00134-014-3325-5.

A. M. Brambilla · E. Prina · F. Nicoli · R. Cosentini $(\bullet)$

Emergency Medicine Department, IRCCS Fondazione Ca' Granda, Ospedale Maggiore Policlinico_Gruppo NIV, Milan, Italy e-mail: r.cosentini@gmail.com

Tel.: +390255033618

S. Aliberti

Department of Health Science, University of Milan Bicocca,

Clinica Pneumologica, AO San Gerardo, Monza, Italy

\section{Del Forno - S. Nava}

Department of Specialistic, Diagnostic and Experimental Medicine, Respiratory and Critical Care Unit, Alma Mater Studiorum,

University of Bologna, Sant'Orsola Malpighi Hospital, Bologna, Italy

G. Ferrari

High Dependency Unit, San Giovanni Bosco Hospital, Piazza

Donatore del Sangue 3, 10154 Turin, Italy
F. Corradi · P. Pelosi IRCCS AOU San Martino IST, Department of Surgical Sciences and Integrated Diagnostics, University of Genoa, Genova, Italy

\section{A. Bignamini}

School of Specialization in Hospital Pharmacy, University of Milan, Via Colombo, 71, Milan, Italy

\section{P. Tarsia}

Department of Pathophysiology and Transplantation, University of Milan IRCCS Fondazione Ca' Granda, Ospedale Maggiore Policlinico, Milan, Italy

\section{Erratum to: Intensive Care Med DOI 10.1007/s00134-014-3325-5}

The name of the fifth author, Manuela Del Forno, was rendered wrongly in her affiliation. The name is shown correctly here. 